\title{
BMR
}

\section{Daidzein promotes osteoblast proliferation and differentiation in OCT1 cells through stimulating the activation of BMP-2/Smads pathway}

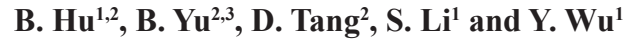 \\ 'Nanchang Second Hospital, Jiangxi University of Traditional Chinese Medicine, \\ Nanchang, China \\ ${ }^{2}$ Longhua Hospital, Shanghai University of Traditional Chinese Medicine, \\ Shanghai, China \\ ${ }^{3}$ Hong-Hui Hospital, Xi' an Jiaotong University College of Medicine, \\ Xi'an, China \\ Corresponding authors: B. Yu / D. Tang \\ E-mail: yubin3600@163.com / dztang702@126.com
}

Genet. Mol. Res. 15 (2): gmr.15028792

Received May 16, 2016

Accepted June 3, 2016

Published June 24, 2016

DOI http://dx.doi.org/10.4238/gmr.15028792

\begin{abstract}
Daidzein, the most widely studied soy phytoestrogen, is not only a potential antiosteoporosis agent owing to its possible osteogenic activity, but also shows anticancer activity. However, the mechanisms through which daidzein affects osteoblast function have not been investigated thoroughly. Here, we show that daidzein stimulated cell proliferation and differentiation of osteoblasts, demonstrated by upregulation of XTT activity, enhancement of alkaline phosphatase (ALP) activity, and upregulation of osteoblast-specific marker genes, including Runt-related transcription factor 2 (Runx2) and Smad1, as well as upregulation of Runx2 and Smad1 protein expression. To determine the mechanisms underlying daidzein's effects on osteoblast differentiation, we first tested the role of daidzein in
\end{abstract}


bone morphogenetic protein (BMP)-2 gene expression in OCT1 cells, and found that it significantly upregulated the expression of BMP-2. Furthermore, it significantly enhanced the phosphorylated protein level of Smad1/5/8 and the protein level of Osterix and increased the activity of $12 x$ SBE-OC-Luc. Finally, we demonstrated that daidzein stimulated Col I, Runx2, and ALP expression, while these effects were significantly blocked by the BMP signaling inhibitor noggin. Together, our data indicate that daidzein acts through stimulating the activation of BMP-2/Smads pathway to promote osteoblast proliferation and differentiation.

Key words: Daidzein; Phytoestrogen; BMP-2; Smad; Osteoblast; Cell signaling

\section{INTRODUCTION}

Aging population leads to increased incidence of osteoporosis, which presents bone loss and microstructure destruction. This disease is caused by an increase in bone resorption and a reduction of bone formation. Bone extracellular matrix is destroyed in patients with osteoporosis. Without the normal structure, the osteoblast was incapable of normal bone remodeling. Although estrogen replacement therapy has been shown to be effective in increasing the activity of osteoblast, it may increase the incidence of uterine and breast cancers according to the report (Christiansen, 1993).

Phytoestrogens raise lots of concern about their potential benefits in the prevention and treatment for osteoporosis. Daidzein, the most widely studied soy phytoestrogen, shows promise as a potential antiosteoporosis agent. Many reports have shown that daidzein has an effect not only on osteogenic activity, but also has anticancer activity (Adjaklyet al., 2013; Strong et al., 2014; Chen et al., 2015). Daidzein-enhanced osteoblast growth might be mediated through the upregulation of BMP expression in primary osteoblast cells (Jia et al., 2003). Daidzein stimulated cell differentiation and mineralization in mouse osteoblastlike MC3T3-E1 cells (Ge et al., 2006). The effects of daidzein on osteoblasts were mediated through the ERbeta pathway (De Wilde et al., 2004; de Wilde et al., 2006). However, in order to fully exploit its antiosteoporosis properties, more studies are needed to better understand and elucidate all the pathways affected by daidzein.

In present study, we found the explicit molecular mechanisms involved in daidzein effect on the function of osteoblast by performing the in vitro experiments with osteoblastic OCT1 cells. Our data indicated that daidzein promoted osteoblast proliferation and differentiation through stimulating the activation of the BMP-2/Smads pathway.

\section{MATERIAL AND METHODS}

\section{Cell cultures}

OCT1 osteoblast precursor cells, derived from the osteocalcin (OC-TAg) promoter SV-40 transgenic mice calvarias, were cultured in low-glucose $\alpha$-MEM medium with $10 \%$ fetal bovine serum as previously described (Bian et al., 2011). 


\section{Cell proliferation assay}

The XTT assay was performed to measure the effects of daidzein on cell proliferation. OCT1 cells were seeded in 96-well culture plates $\left(1 \times 10^{4}\right.$ cells/well). After cultured for $24 \mathrm{~h}$, cells were intervened with different concentrations $(1,5,10,30$, and $60 \mu \mathrm{g} / \mathrm{mL})$ of daidzein or BMP-2 $(50 \mathrm{ng} / \mathrm{mL})$, with control groups, for $48 \mathrm{~h}$. XTT testing solution, which was prepared by mixing $5 \mathrm{~mL}$ XTT-labeling reagents (Sigma, USA) with $100 \mu \mathrm{L}$ electron coupling reagents, was added to each well. After incubating for $4 \mathrm{~h}$, we used an ELISA Plate reader (Thermo Electron Corporation, Waltham, MA, USA) to detect the absorbance at a test wavelength of $492 \mathrm{~nm}$ and a reference wavelength of $690 \mathrm{~nm}$.

\section{Assay of ALP activity}

Cells cultured on 96-well culture plates $\left(1 \times 10^{4}\right.$ cells/well $)$ for $24 \mathrm{~h}$, were treated with different concentrations $(1,5,10,30$, and $60 \mu \mathrm{g} / \mathrm{mL})$ of daidzein or BMP-2 $(50 \mathrm{ng} / \mathrm{mL})$, including controls, for $48 \mathrm{~h}$. Then we washed cells for three times using $0.9 \% \mathrm{NaCl}$ buffer and collected the cell lysates with M-Per (Pierce, Rockford, IL, USA). Substrate p-nitrophenol phosphate was prepared by dissolving in AMP buffer (Bio-Rad, Hercules, CA, USA). Total lysates $(20 \mu \mathrm{L})$ were added to $1 \mathrm{~mL}$ substrate. After incubating for $5 \mathrm{~min}$, we added $0.5 \mathrm{~mL}$ stop solution $\left(0.3 \mathrm{M} \mathrm{Na}_{3} \mathrm{PO}_{4}\right)$ to the reaction system and used an ELISA Plate reader (Thermo Electron Corporation) to detect the absorbance at a test wavelength of $410 \mathrm{~nm}$ for the measure of ALP activity. We normalized the absorbance values to the total protein levels which were quantified by using a BCA kit (Pierce).

\section{ALP staining assay}

After incubation for $48 \mathrm{~h}$ with or without daidzein $(10 \mu \mathrm{g} / \mathrm{mL})$ and noggin $(300 \mathrm{ng} /$ $\mathrm{mL}$ ), cells were fixed with $10 \%$ neutral buffered formalin for $15 \mathrm{~min}$ and washed cells by using $0.9 \% \mathrm{NaCl}$ buffer for three times. Then cells were stained at $37^{\circ} \mathrm{C}$ for $30 \mathrm{~min}$ by using the NBT-BCIP buffer (Bio-Rad).

\section{Transfection and luciferase activity assay}

For the luciferase activity assay, we transfected the constructs of $12 \mathrm{xSBE}-\mathrm{OC}$ into OCT1 cells by using FUGENE HD reagents. We also co-transfected an SV40-Renilla luciferase construct with the above reporter construct to normalize the result for detecting the efficiency of transfection. Then cells were continued to culture for $48 \mathrm{~h}$ with or without daidzein $(10 \mu \mathrm{g} /$ $\mathrm{mL})$ and noggin $(300 \mathrm{ng} / \mathrm{mL})$. Cell lysates were extracted after treatment for $48 \mathrm{~h}$, and the activity of luciferase was detected by using a Promega luciferase assay kit. Luciferase activity in the cell lysate was determined using a luminometer (Thermo Electron Corporation).

\section{Real-time quantitative polymerase chain reaction (qPCR) analysis}

Total cellular RNA was isolated from cultured OCT1 cells after $48 \mathrm{~h}$-treatment with or without different concentrations $(1,5,10,30$ and $60 \mu \mathrm{g} / \mathrm{mL})$ of daidzein or BMP-2 (50 ng/ $\mathrm{mL})$ or noggin $(300 \mathrm{ng} / \mathrm{mL})$. Total RNA was extracted using the Qiagen RNA easy mini kit 
(Valencia, CA, USA) and the first-strand cDNA was synthesized using $1 \mu \mathrm{g}$ of RNA with a Clontech RT kit primed by an oligo primer (Mountain View, CA, USA). The qPCR analysis was performed with mouse specific primers, which were listed in Table 1. The expression levels of these genes were normalized to $\beta$-actin.

Table 1. Mouse primers for qPCR assays.
\begin{tabular}{l|l|l}
\hline Genes & Forward primers & Reverse primers \\
\hline$\beta$-actin & CTGTCCCTGTATGCCTCTG & ATGTCACGCACGATTTCC \\
\hline BMP-2 & GATCTGTACCGCAGGCACTC & TTCCCACTCATCTCTGGAAGTT \\
\hline Runx2 & CATTTGCACTGGGTCACACGTA & GAATCTGGCCATGTTTGTGCTC \\
\hline Smad1 & CACCTGCTTACCTGCCTCCT & TGCCTGAACATCTCCTCTGCT \\
\hline Col I & TGATCACTCCCACGTTTTCA & CTGGGCCTGGTAGTTGTTGT \\
\hline ALP & TGACCTTCTCTCCTCCATCC & CTTCCTGGGAGTCTCATCCT \\
\hline
\end{tabular}

\section{Western blot analysis}

Total cellular protein was extracted from cultured OCT1 cells after treatment with different concentrations $(10 \mu \mathrm{g} / \mathrm{mL})$ of daidzein or BMP-2 $(50 \mathrm{ng} / \mathrm{mL})$ or noggin $(300 \mathrm{ng} /$ $\mathrm{mL}$ ), and controls, for 48 or $2 \mathrm{~h}$. Cells were lysed using protein isolation buffer, including $150 \mathrm{mM}$ sodium chloride, $50 \mathrm{mM}$ TRIS, $10 \%$ protease inhibitor cocktail, and 1\% IGEPAL. We then used a BCA protein assay kit (Pierce) to quantify. Proteins were fractionated by SDS-polyacrylamide gel electrophoresis, transferred to a nitrocellulose membrane, incubated with anti-Runx2, anti-Osx (Cell signaling Tech, USA), anti-p-Smad1/5/8, anti-Smad1, and anti- $\beta$-actin (Oncogene Research Products, San Diego, CA, USA) antibodies overnight at $4^{\circ} \mathrm{C}$. After washing, membranes were incubated with secondary HRP-conjugated antibodies (Jackson Immunoresearch) for $1 \mathrm{~h}$ at room temperature. Bands were detected by enhanced chemiluminescence-mediated visualization kit (Amersham Biosciences, Piscataway, NJ, USA).

\section{Statistical analysis}

All quantitative experiments were repeated three times independently, and the data are reported as means \pm standard deviation. We performed the one-way ANOVA analysis for multiple-group comparison, and the unpaired Student $t$-test for two-group comparison. P value less than 0.05 was considered as significant.

\section{RESULTS}

\section{Daidzein promotes osteoblast proliferation dose-dependently}

We examined the role of daidzein in the proliferation of OCT1 cells by the assay of XTT. We found that daidzein promoted the proliferation of osteoblastic cells dose-dependently, with a maximal role at the dose of $10 \mu \mathrm{g} / \mathrm{mL}$ after 48 -h treatment $(\mathrm{P}<0.05$, Figure 1$)$.

\section{Daidzein enhances osteoblast differentiation dose-dependently}

To test if daidzein also plays a role in the differentiation of osteoblast, ALP activity assay, RT-PCR assay and Western-blot assay were performed. Because ALP is a key marker 
for the differentiation of osteoblast, we firstly examined the activity of ALP. ALP activity was shown a significant increase after the treatment of daidzein in a dose-dependent manner, with a maximal role at a dose of $10 \mu \mathrm{g} / \mathrm{mL}(\mathrm{P}<0.05)$ in OCT1 cells (Figure 2A). The expression of osteoblastic marker genes such as Runx2 $(\mathrm{P}<0.05)$ and Smad1 $(\mathrm{P}<0.05)$ were also shown a remarkable increase the treatment of daidzein at a concentration of $10 \mu \mathrm{g} / \mathrm{mL}$ (Figure 2B-C). We also found that daidzein significantly increased the protein levels of Runx2 and Smad1 at a concentration of $10 \mu \mathrm{g} / \mathrm{mL}$ (Figure 2D). Together, our data indicate that daidzein enhances the differentiation of osteoblast.

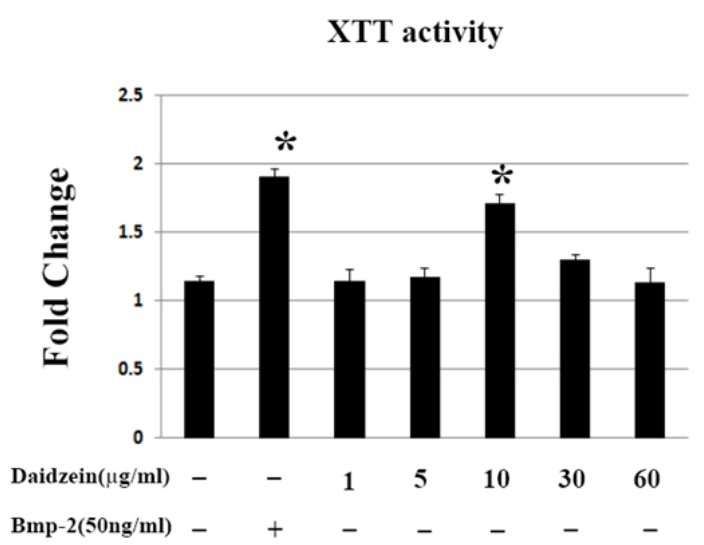

Figure 1. Daidzein promoted osteoblast proliferation dose-dependently. XTT assay showed that daidzein significantly promoted the proliferation of osteoblastic OCT1cells at a concentration of $10 \mu \mathrm{g} / \mathrm{mL}$ after $48-\mathrm{h}$ treatment. ${ }^{*} \mathrm{P}<0.05$, compared with control.
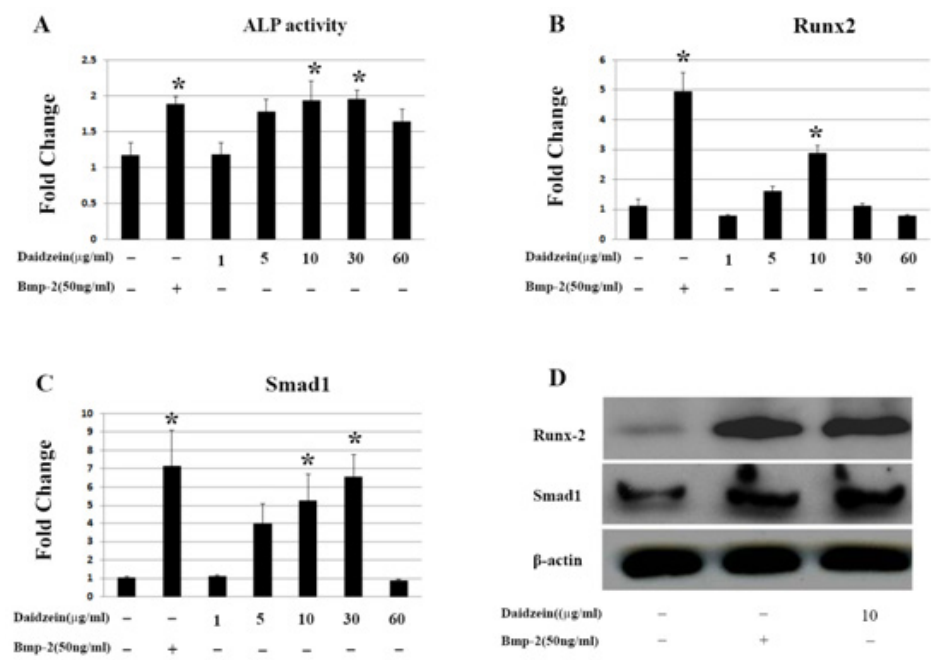

Figure 2. Daidzein stimulated osteoblast differentiation dose-dependently. A. Daidzein significantly enhanced ALP activity in OCT1 cells after 48-h treatment. B. Daidzein significantly increased Runx2 gene expression in OCT1 cells after 48-h treatment. C. Daidzein significantly increased Smad1 gene expression in OCT1 cells after 48-h treatment. D. Daidzein increased the protein levels of Runx2 and Smad1 in OCT1 cells after 48-h treatment. ${ }^{*} \mathrm{P}<$ 0.05 , compared with control. 


\section{Daidzein activates BMP-2/Smads pathway}

To make the mechanism of daidzein clear on the differentiation of osteoblast, we first detected the role of daidzein in the expression of BMP-2 gene in OCT1 cells. We showed that daidzein significantly upregulated the gene expression of BMP-2 $(\mathrm{P}<0.05)$, particularly at a concentration of $10 \mu \mathrm{g} / \mathrm{mL}$, which showed a 12.7 -fold increase (Figure $3 \mathrm{~A}$ ). We then treated cells for only $2 \mathrm{~h}$ and examined the effect of daidzein $(10 \mu \mathrm{g} / \mathrm{mL})$ on the Smad1/5/8 proteins, the key molecules of BMP pathway. Although we did not find any difference in the total Smad1/5/8 protein levels, we did find that daidzein significantly upregulated the phosphorylated protein levels of Smad1/5/8, while its effect was significantly blocked by noggin, a specific BMP signaling inhibitor (Figure 3B). We also tested the role of daidzein in the protein level of osterix (Osx), which is a direct target gene of BMP pathway. The result showed that daidzein significantly increased Osx protein level by quantified analysis $(\mathrm{P}<0.05)$. However, its effect was significantly blocked by noggin $(\mathrm{P}<0.05)$ (Figure $3 \mathrm{C}, \mathrm{D})$. Finally, to further determine the role of daidzein in BMP pathway, we detected the efficacy of daidzein on the $12 x$ SBE-OC-Luc activity, which represents the activity of a BMP signaling reporter. We found that daidzein enhanced the $12 \mathrm{xSBE}-\mathrm{OC}$-Luc activity with a 3.1 -fold increase in OCT1 cells $(\mathrm{P}<0.05)$, while its efficacy was significantly eliminated by noggin $(\mathrm{P}<0.05)$ (Figure $3 \mathrm{E})$. Together, our data demonstrate that daidzein may activate BMP-2/Smads pathway.
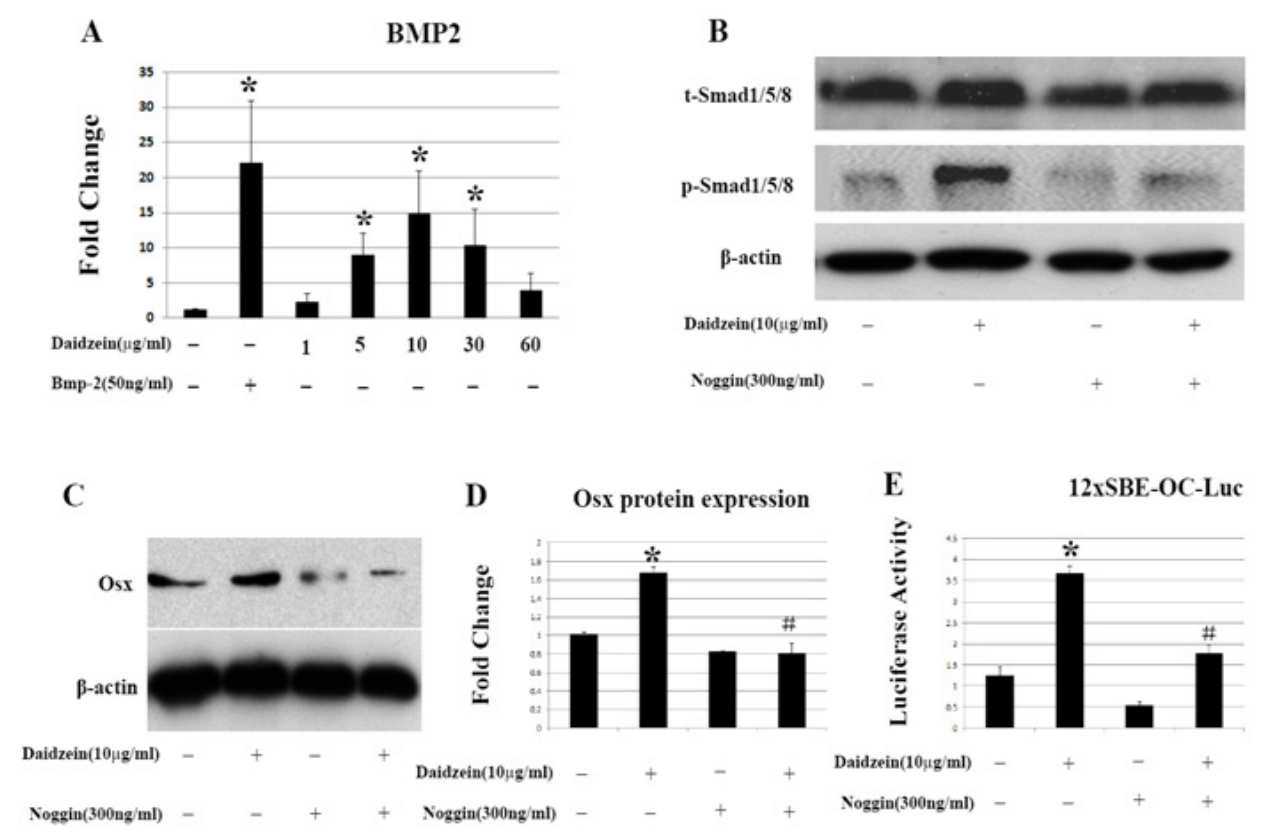

D

Osx protein expression

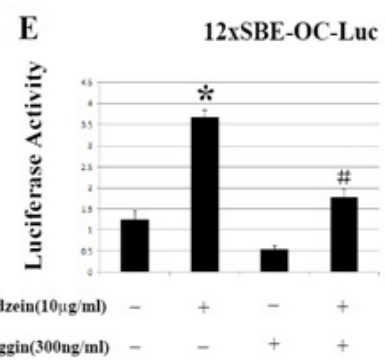

Figure 3. Daidzein activated BMP-2/Smads signaling. A. Daidzein significantly upregulated BMP-2mRNA expression in OCT1 cells after 48-h treatment. B. Daidzein significantly enhanced the expression of phosphorylated Smad1/5/8 protein in OCT 1 cells after 2 -h treatment, and its effect was significantly blocked by noggin. C. Daidzein significantly increased the protein level of Osx expression in OCT1 cells after 48-h treatment, and its effect was significantly blocked by noggin. D. The quantitative data of C. E. Daidzein significantly increased the activity of 12xSBE-OC-Luc in OCT1 cells after 48 -h treatment, and its effect was significantly blocked by noggin. $* \mathrm{P}<0.05$, compared with control; ${ }^{\mathrm{P}}<0.05$, compared with the group of daidein. 


\section{Daidzein-enhanced osteoblast proliferation and differentiation is mediated through stimulating the activation of BMP-2/Smads pathway}

To further make clear whether daidzein-enhanced osteoblast proliferation and differentiation occurs via BMP-2/Smads pathway, we first performed an XTT assay to test the effect of daidzein $(10 \mu \mathrm{g} / \mathrm{mL})$ on cell proliferation with noggin, a specific inhibitor of BMP signaling. We found that daidzein significantly stimulated osteoblast proliferation $(\mathrm{P}<0.05)$, however its effect was significantly eliminated by noggin $(\mathrm{P}<0.05)$ (Figure $4 \mathrm{~A})$. We next detected the roles of daidzein $(10 \mu \mathrm{g} / \mathrm{mL})$ in the expression of some osteoblastic marker genes with or without co-treatment of noggin. We found that daidzein remarkably increased the mRNA level of Type I collagen in osteoblasts with a 9.9-fold change $(\mathrm{P}<0.05)$, while its role was significantly eliminated by noggin $(\mathrm{P}<0.05)$ (Figure $4 \mathrm{~B})$. We also found a significant increase in the mRNA level of Runx2 expression in osteoblasts with a 2.9-fold change $(\mathrm{P}<0.05)$ after the treatment of daidzein, which effect that was also remarkably eliminated by noggin $(\mathrm{P}<0.05)$ (Figure $4 \mathrm{C})$. We also found that daidzein significantly upregulated ALP gene expression in osteoblasts with an 8.1-fold increase $(\mathrm{P}<0.05)$, and this effect was also eliminated by noggin $(\mathrm{P}<0.05)$ (Figure 4D). Finally, ALP staining data showed that daidzein significantly enhanced ALP expression in osteoblasts, and this effect was significantly reversed by noggin (Figure 4E). These findings indicate that daidzein induces osteoblast proliferation and differentiation through stimulating the activation of the BMP-2/Smads pathway, and that a BMP signaling inhibitor can block these effects.

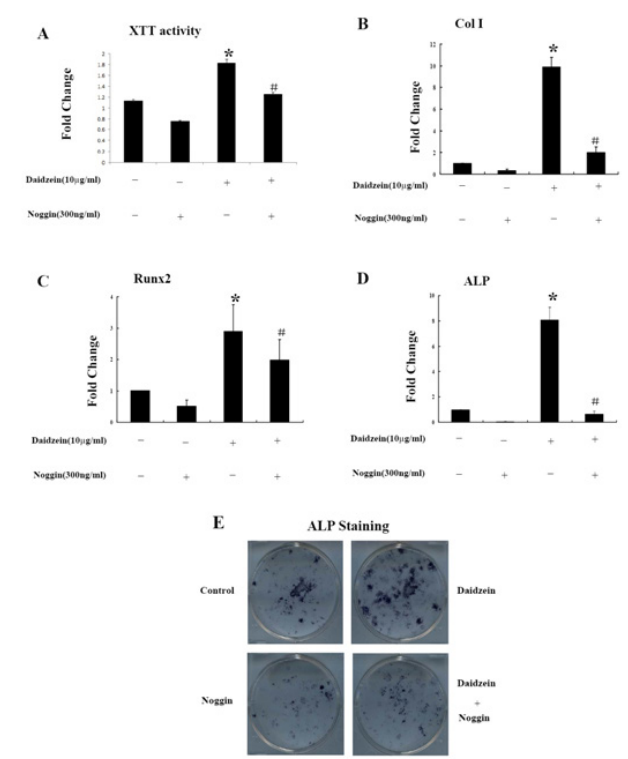

Figure 4. Daidzein-induced osteoblast proliferation and differentiation was mediated through simulating the activation of the BMP-2/Smads signaling. A. Daidzein significantly increased the XTT activity in OCT1 cells after 48-h treatment, and its effect was significantly blocked by noggin. B. Daidzein significantly increased mRNA expression of Col I in OCT1 cells after 48-h treatment, and its effect was significantly blocked by noggin. C. Daidzein significantly increased mRNA expression of Runx2 in OCT1 cells after 48-h treatment, and its effect was significantly blocked by noggin. D. Daidzein significantly increased mRNA expression of ALP in OCT1 cells after 48-h treatment, and its effect was significantly blocked by noggin. E. ALP staining results showed that daidzein significantly enhanced ALP expression in OCT1 cells after 48-h treatment, and its effect was significantly blocked by noggin. ${ }^{*} \mathrm{P}<0.05$, compared with control; ${ }^{*} \mathrm{P}<0.05$, compared with the group of daidein. 


\section{DISCUSSION}

Daidzein is present in a number of plants and herbs such as Pueraria mirifica and Pueraria lobata. Previous studies have shown that daidzein has an anti-osteoporosis effect. Jia et al. (2003) found that daidzein increased the viability of osteoblasts, ALP activity, osteocalcin synthesis and BMP-2 expression in primary osteoblastic cells. Strong et al. (2014) reported that daidzein enhanced the expression of some ostegenic genes in human bone marrow-derived mesenchymal stem cells (HBMSCs), such as Osx, ALP, and OPN. The stimulatory role of daidzein on the osteoblast differentiation and mineralization in MC3T3-E1 cells has also been reported (Ge et al. 2006). In this study, we show that daidzein stimulated osteoblast proliferation and differentiation in OCT1 cells. Treatment of osteoblastic cells with daidzein increased the activity of cell proliferation and the expression of some osteogenic differentiation marker genes, such as ALP, Smad1, Runx2, Osx, Col I, etc. It has been reported that the effect of daidein on osteoblast differentiation was mediated by the expression of BMP-2.However, the detailed molecular mechanism remains unknown (Jia et al., 2003).

It is well known that BMPs are very important for the cell proliferation and differentiation of osteoblast (Sykaras and Opperman, 2003). BMP-2 binds to their cell surface receptors, and subsequently induces the phosphorylation of Smad1/5/8, then acts on bone cells. Phosphorylated Smad1/5/8 and Smad4 forms a protein complex, translocates into the nucleus to activate the bone-specific-genes transcription, which in turn promote osteoblast differentiation (Sykaras and Opperman, 2003; Chen et al., 2004; Miyazono et al., 2005; Wan and Cao, 2005; Bilican et al., 2008). As a matter of fact, it has been reported that several small molecular weight compounds can be able to activate BMP-2 signaling (Mundy et al., 1999; Garrett et al., 2003; Tang et al., 2010; Tang et al., 2011; Li et al., 2013). To clarify the mechanism by which daidzein promotes the cell proliferation and differentiation of osteoblast, we tested if it can activate BMP-2 signaling. First, we found that the intervention of osteoblastic cells with daidzein increased the gene expression of BMP-2. Second, we found that daidzein increased the phosphorylated protein level of Smad1/5/8, the key molecules in BMP pathway, as well as the gene expression of Osx, a direct target gene of BMP signaling. We further demonstrated that daidzein increased the $12 x$ SBE-OC-Luc activity, which is a BMP signaling reporter. Moreover, our data also showed that daidzein-activated BMP signaling could be blocked by the use of noggin, a specific BMP signaling inhibitor.

Further investigations were carried out to confirm whether daidzein-promoted osteoblast differentiation is mediated by BMP signaling. We found that noggin, a specific BMP signaling inhibitor, blocked a daidzein-induced increase in gene expression of Col I, Runx2, and ALP, suggesting that daidzein promotes the differentiation of osteoblast through activating the BMP signaling pathway.

In addition to effecting osteoblasts, daidzein was also reported to inhibit osteoclast differentiation by increasing ERalpha expression (Garcia Palacios et al., 2005). Here, we did not investigate the efficacy of daidzein on osteoclasts, focusing instead only on its effects on osteoblasts. In future studies, we will analyze the effects and detailed molecular mechanisms of daidzein on osteoclastogenesis.

In conclusion, our findings demonstrate that daidzein promotes osteoblast proliferation and differentiation by stimulating the activation of the BMP-2/Smads signaling. 


\section{Conflicts of interest}

The authors declare no conflict of interest.

\section{ACKNOWLEDGMENTS}

Research supported in part by the NSFC Program (\#81473701), the Shanghai RisingStar Program (\#14QA1403500) and the Outstanding Young Training Plan in Shanghai Health System (\#XYQ2013085).

\section{REFERENCES}

Adjakly M, Ngollo M, Boiteux JP, Bignon YJ, et al. (2013). Genistein and daidzein: different molecular effects on prostate cancer. Anticancer Res. 33: 39-44.

Bian Q, Huang JH, Liang QQ, Shu B, et al. (2011). The osteogenetic effect of astragaloside IV with centrifugating pressure on the OCT-1 cells. Pharmazie 66: 63-68.

Bilican B, Fiore-Heriche C, Compston A, Allen ND, et al. (2008). Induction of Olig2 precursors by FGF involves BMP signalling blockade at the Smad level. PLoS One 3: e2863. http://dx.doi.org/10.1371/journal.pone.0002863

Chen D, Zhao M and Mundy GR (2004). Bone morphogenetic proteins. Growth Factors 22: 233-241. http://dx.doi.org/1 $\underline{0.1080 / 08977190412331279890}$

Chen Y, Cass SL, Kutty SK, Yee EM, et al. (2015). Synthesis, biological evaluation and structure-activity relationship studies of isoflavene based Mannich bases with potent anti-cancer activity. Bioorg. Med. Chem. Lett. 25: 5377-5383. http://dx.doi.org/10.1016/j.bmcl.2015.09.027

Christiansen C (1993). Consensus development conference: diagnosis, prophylaxis, and treatment of osteoporosis. Am. J. Med. 94: 646-650. http://dx.doi.org/10.1016/0002-9343(93)90218-E

de Wilde A, Lieberherr M, Colin C and Pointillart A (2004). A low dose of daidzein acts as an ERbeta-selective agonist in trabecular osteoblasts of young female piglets. J. Cell. Physiol. 200: 253-262. http://dx.doi.org/10.1002/jcp.20008

de Wilde A, Heberden C, Chaumaz G, Bordat C, et al. (2006). Signaling networks from Gbeta1 subunit to transcription factors and actin remodeling via a membrane-located ERbeta-related protein in the rapid action of daidzein in osteoblasts. J. Cell. Physiol. 209: 786-801. http://dx.doi.org/10.1002/jcp.20767

Garcia Palacios V, Robinson LJ, Borysenko CW, Lehmann T, et al. (2005). Negative regulation of RANKL-induced osteoclastic differentiation in RAW264.7 Cells by estrogen and phytoestrogens. J. Biol. Chem. 280: 13720-13727. http://dx.doi.org/10.1074/jbc.M410995200

Garrett IR, Chen D, Gutierrez G, Zhao M, et al. (2003). Selective inhibitors of the osteoblast proteasome stimulate bone formation in vivo and in vitro. J. Clin. Invest. 111: 1771-1782. http://dx.doi.org/10.1172/JCI16198

Ge Y, Chen D, Xie L and Zhang R (2006). Enhancing effect of daidzein on the differentiation and mineralization in mouse osteoblast-like MC3T3-E1 cells. Yakugaku Zasshi 126: 651-656. http://dx.doi.org/10.1248/yakushi.126.651

Jia TL, Wang HZ, Xie LP, Wang XY, et al. (2003). Daidzein enhances osteoblast growth that may be mediated by increased bone morphogenetic protein (BMP) production. Biochem. Pharmacol. 65: 709-715. http://dx.doi. org/10.1016/S0006-2952(02)01585-X

Li XF, Xu H, Zhao YJ, Tang DZ, et al. (2013). Icariin Augments Bone Formation and Reverses the Phenotypes of Osteoprotegerin-Deficient Mice through the Activation of Wnt/ $\beta$-Catenin-BMP Signaling. Evid. Based Complement. Alternat. Med. 2013: 652317.

Miyazono K, Maeda S and Imamura T (2005). BMP receptor signaling: transcriptional targets, regulation of signals, and signaling cross-talk. Cytokine Growth Factor Rev. 16: 251-263. http://dx.doi.org/10.1016/j.cytogfr.2005.01.009

Mundy G, Garrett R, Harris S, Chan J, et al. (1999). Stimulation of bone formation in vitro and in rodents by statins. Science 286: 1946-1949. http://dx.doi.org/10.1126/science.286.5446.1946

Strong AL, Jiang Q, Zhang Q, Zheng S, et al. (2014). Design, synthesis, and osteogenic activity of daidzein analogs on human mesenchymal stem cells. ACS Med. Chem. Lett. 5: 143-148. http://dx.doi.org/10.1021/ml400397k

Sykaras N and Opperman LA (2003). Bone morphogenetic proteins (BMPs): how do they function and what can they offer the clinician? J. Oral Sci. 45: 57-73. http://dx.doi.org/10.2334/josnusd.45.57

Tang DZ, Hou W, Zhou Q, Zhang M, et al. (2010). Osthole stimulates osteoblast differentiation and bone formation by activation of $\beta$-catenin-BMP signaling. J. Bone Miner. Res. 25: 1234-1245. http://dx.doi.org/10.1002/jbmr.21

Genetics and Molecular Research 15 (2): gmr.15028792

CFUNPEC-RP www.funpecrp.com.br 
Tang DZ, Yang F, Yang Z, Huang J, et al. (2011). Psoralen stimulates osteoblast differentiation through activation of BMP signaling. Biochem. Biophys. Res. Commun. 405: 256-261. http://dx.doi.org/10.1016/j.bbrc.2011.01.021

Wan M and Cao X (2005). BMP signaling in skeletal development. Biochem. Biophys. Res. Commun. 328: 651-657. http:// dx.doi.org/10.1016/j.bbrc.2004.11.067 